\title{
Differential equations on complex projective hypersurfaces of low dimension
}

\author{
Simone Diverio
}

\begin{abstract}
Let $n=2,3,4,5$ and let $X$ be a smooth complex projective hypersurface of $\mathbb{P}^{n+1}$. In this paper we find an effective lower bound for the degree of $X$, such that every holomorphic entire curve in $X$ must satisfy an algebraic differential equation of order $k=n=\operatorname{dim} X$, and also similar bounds for order $k>n$. Moreover, for every integer $n \geqslant 2$, we show that there are no such algebraic differential equations of order $k<n$ for a smooth hypersurface in $\mathbb{P}^{n+1}$.
\end{abstract}

\section{Introduction}

Let $X \subset \mathbb{P}^{n+1}$ be a complex projective hypersurface, with $\operatorname{deg} X=d$. In 1970 Kobayashi conjectured [Kob70] that $X$ is hyperbolic provided that $X$ is generic and $d$ is large enough. During recent years, efforts have been made to treat both the low-dimension cases (with special attention paid to the lower bound for the degree) and the general case.

For instance, [DEG00] proved the conjecture for very generic surfaces in $\mathbb{P}^{3}$ of degree greater than or equal to 21, [Rou07] proved a weaker form (namely weak analytic hyperbolicity) for generic hypersurfaces in $\mathbb{P}^{4}$ of degree greater than or equal to 593, and [Siu04] announced a complete proof of the Kobayashi conjecture in any dimension, for $d \gg n$.

The major technique in the study of the Kobayashi hyperbolicity question is probably to analyse the existence of sections of the bundle $E_{k, m}^{\mathrm{GG}} T_{X}^{*}$ of differentials of order $k$ and weighted degree $m$, as defined in [GG79]. This technique was later refined by Demailly in [Dem97], with the introduction of the bundle $E_{k, m} T_{X}^{*}$ of invariant differential operators.

Let $X$ be a compact complex manifold. Then $X$ is Kobayashi hyperbolic if and only if there are no non-constant entire holomorphic curves in $X$ (Brody's criterion). The general philosophy is that global holomorphic sections of $E_{k, m} T_{X}^{*}$ vanishing on a fixed ample divisor give rise to global algebraic differential equations that every entire holomorphic curve must satisfy.

It is known from [DEG00] that every smooth surface in $\mathbb{P}^{3}$ of degree greater than or equal to 15 has such differential equations of order two. For the dimension three case [Rou06b] observed that one needs to look for order three equations since one has in general the vanishing of symmetric differentials and invariant 2-jet differentials for smooth hypersurfaces in projective 4-space. On the other hand [Rou06b] shows the existence of global invariant 3-jet differentials vanishing on an ample divisor on every smooth hypersurface $X$ in $\mathbb{P}^{4}$, provided that $\operatorname{deg} X \geqslant 97$.

The existence of these global sections is shown by means of a delicate algebraic study of the bundle $E_{3, m} T_{X}^{*}$ conducted in [Rou06a], which permits one to compute the Euler characteristic. Then by a laborious estimate of the dimension of the higher cohomology groups, one can find a positive lower bound for the dimension of the space of global sections (at least for $m$ large).

Received 23 July 2007, accepted in final form 26 November 2007.

2000 Mathematics Subject Classification 14F99, 32Q45.

Keywords: Kobayashi hyperbolicity, invariant jet differentials, Schur powers, holomorphic Morse inequalities.

This journal is (C) Foundation Compositio Mathematica 2008. 


\section{DifFERENTIAL EQUATIONS ON COMPLEX PROJECTIVE HYPERSURFACES}

TABLE 1. Effective lower bounds for degree $d$.

\begin{tabular}{rrrrrr}
\hline & \multicolumn{5}{c}{$k$} \\
\cline { 2 - 5 }$n$ & 1 & 2 & 3 & \multicolumn{1}{c}{4} & \multicolumn{1}{c}{5} \\
\hline 2 & & 18 & 16 & 16 & 16 \\
3 & & & 82 & 74 & 74 \\
4 & & & & 329 & 298 \\
5 & & & & & 1222 \\
\hline
\end{tabular}

In the present paper, we first of all generalize to all dimensions the non-existence of global sections of invariant jet differentials of order less than the dimension of the ambient variety. In fact, we prove the following theorem.

Theorem 1. Let $X \subset \mathbb{P}^{n+1}$ be a smooth hypersurface. Then

$$
H^{0}\left(X, E_{k, m} T_{X}^{*}\right)=0
$$

for all $m \geqslant 1$ and $1 \leqslant k \leqslant n-1$. In other words, on a smooth projective hypersurface there are no global invariant jet differentials of order less than its dimension.

The idea of the proof is to exclude, in the direct sum decomposition into irreducible $\operatorname{Gl}\left(T_{X}^{*}\right)$ representation of the graded bundle $\mathrm{Gr}^{\bullet} E_{k, m} T_{X}^{*}$, the existence of Schur powers $\Gamma^{\left(\lambda_{1}, \ldots, \lambda_{n}\right)} T_{X}^{*}$, with $\lambda_{n}>0$, and then to use a vanishing theorem due to Brückmann and Rackwitz [BR90] for Schur powers of the cotangent bundle of smooth projective complete intersections.

On the other hand, in the direction of existence of global invariant jet differentials, we get a slightly better bound for smooth hypersurfaces in $\mathbb{P}^{4}$ and a new result for smooth hypersurfaces in $\mathbb{P}^{5}$ and $\mathbb{P}^{6}$.

Theorem 2. Let $X \subset \mathbb{P}^{n+1}, n=2,3,4,5$, be a smooth hypersurface of degree $d$ and $A \rightarrow X$ an ample line bundle. Then

$$
H^{0}\left(X, E_{k, m} T_{X}^{*} \otimes A^{-1}\right) \neq 0
$$

for $m$ large enough and $k \geqslant n$ if $d$ is sufficiently big, and therefore every holomorphic entire curve $f: \mathbb{C} \rightarrow X$ must satisfy the corresponding algebraic differential equation.

Moreover, we have the effective lower bounds for the degree $d$ as shown in Table 1 (depending on the values of $n$ and $k$ ).

Here the proof is achieved without any Euler characteristic computation, thanks to the algebraic version [Tra95] of Demailly's holomorphic Morse inequalities. In our case, the Morse inequalities are applied to a particular subalgebra of $E_{k, m} T_{X}^{*}$ which is easier to compute than the full algebra $\bigoplus_{m} E_{k, m} T_{X}^{*}$ itself - although its positivity properties are probably not as good.

We would like to point out that in this way no higher cohomology computations nor any algebraic study of the jet bundle are needed. However, even if a priori these techniques should work in higher dimension, the amount of computations needed to get the result blows up rapidly when the dimension increases.

\section{Background material and preliminaries}

In this section, we follow [Dem97] very closely. 


\section{S. DIVERIO}

\subsection{Jet differentials}

Let $(X, V)$ be a directed manifold, i.e. a pair where $X$ is a complex manifold and $V \subset T_{X}$ a holomorphic subbundle (not necessarily integrable) of the tangent bundle. The bundle $J_{k} V$ is the bundle of $k$-jets of holomorphic curves $f:(\mathbb{C}, 0) \rightarrow X$ which are tangent to $V$, i.e. such that $f^{\prime}(t) \in V_{f(t)}$ for all $t$ in a neighbourhood of 0 , together with the projection map $f \mapsto f(0)$ onto $X$.

Let $\mathbb{G}_{k}$ be the group of germs of $k$-jets of biholomorphisms of $(\mathbb{C}, 0)$, that is, the group of germs of biholomorphic maps

$$
t \mapsto \varphi(t)=a_{1} t+a_{2} t^{2}+\cdots+a_{k} t^{k}, \quad a_{1} \in \mathbb{C}^{*}, a_{j} \in \mathbb{C}, j \geqslant 2,
$$

in which the composition law is taken modulo terms $t^{j}$ of degree $j>k$. Then $\mathbb{G}_{k}$ admits a natural fibrewise right action on $J_{k} V$ consisting of reparametrizing $k$-jets of curves by a biholomorphic change of parameter. Moreover the subgroup $\mathbb{H} \simeq \mathbb{C}^{*}$ of homotheties $\varphi(t)=\lambda t$ is a (non-normal) subgroup of $\mathbb{G}_{k}$ and we have a semidirect decomposition $\mathbb{G}_{k}=\mathbb{G}_{k}^{\prime} \ltimes \mathbb{H}$, where $\mathbb{G}_{k}^{\prime}$ is the group of $k$-jets of biholomorphisms tangent to the identity. The corresponding action on $k$-jets is described in coordinates by

$$
\lambda \cdot\left(f^{\prime}, f^{\prime \prime}, \ldots, f^{(k)}\right)=\left(\lambda f^{\prime}, \lambda^{2} f^{\prime \prime}, \ldots, \lambda^{k} f^{(k)}\right) .
$$

As in [GG79], we introduce the vector bundle $E_{k, m}^{\mathrm{GG}} V^{*} \rightarrow X$ whose fibres are complex valued polynomials $Q\left(f^{\prime}, f^{\prime \prime}, \ldots, f^{(k)}\right)$ on the fibres of $J_{k} V$, of weighted degree $m$ with respect to the $\mathbb{C}^{*}$ action defined by $\mathbb{H}$, that is, such that

$$
Q\left(\lambda f^{\prime}, \lambda^{2} f^{\prime \prime}, \ldots, \lambda^{k} f^{(k)}\right)=\lambda^{m} Q\left(f^{\prime}, f^{\prime \prime}, \ldots, f^{(k)}\right),
$$

for all $\lambda \in \mathbb{C}^{*}$ and $\left(f^{\prime}, f^{\prime \prime}, \ldots, f^{(k)}\right) \in J_{k} V$.

Next, we define the bundle of Demailly-Semple jet differentials (or invariant jet differentials) as a subbundle of the Green-Griffiths one.

Definition 1 [Dem97]. The bundle of invariant jet differentials of order $k$ and degree $m$ is the subbundle $E_{k, m} V^{*} \subset E_{k, m}^{\mathrm{GG}} V^{*}$ of polynomial differential operators $Q\left(f^{\prime}, f^{\prime \prime}, \ldots, f^{(k)}\right)$ which are invariant under arbitrary changes of parametrization, i.e. for every $\varphi \in \mathbb{G}_{k}$

$$
Q\left((f \circ \varphi)^{\prime},(f \circ \varphi)^{\prime \prime}, \ldots,(f \circ \varphi)^{(k)}\right)=\varphi^{\prime}(0)^{m} Q\left(f^{\prime}, f^{\prime \prime}, \ldots, f^{(k)}\right) .
$$

Alternatively, $E_{k, m} V^{*}=\left(E_{k, m}^{\mathrm{GG}} V^{*}\right)^{\mathbb{G}_{k}^{\prime}}$ is the set of invariants of $E_{k, m}^{\mathrm{GG}} V^{*}$ under the action of $\mathbb{G}_{k}^{\prime}$.

We now define a filtration on $E_{k, m}^{\mathrm{GG}} V^{*}$. A coordinate change $f \mapsto \Psi \circ f$ transforms every monomial $\left(f^{(\bullet)}\right)^{\ell}=\left(f^{\prime}\right)^{\ell_{1}}\left(f^{\prime \prime}\right)^{\ell_{2}} \cdots\left(f^{(k)}\right)^{\ell_{k}}$ of partial weighted degree $|\ell|_{s}:=\ell_{1}+2 \ell_{2}+\cdots+s \ell_{s}, 1 \leqslant s \leqslant k$, into a polynomial $\left((\Psi \circ f)^{(\bullet)}\right)^{\ell}$ in $\left(f^{\prime}, f^{\prime \prime}, \ldots, f^{(k)}\right)$, which has the same partial weighted degree of order $s$ if $\ell_{s+1}=\cdots=\ell_{k}=0$ and a larger or equal partial degree of order $s$ otherwise. Hence, for each $s=1, \ldots, k$, we get a well defined decreasing filtration $F_{s}^{\bullet}$ on $E_{k, m}^{\mathrm{GG}} V^{*}$ as follows:

$$
F_{s}^{p}\left(E_{k, m}^{\mathrm{GG}} V^{*}\right)=\left\{\begin{array}{c}
Q\left(f^{\prime}, f^{\prime \prime}, \ldots, f^{(k)}\right) \in E_{k, m}^{\mathrm{GG}} V^{*} \text { involving } \\
\text { only monomials }\left(f^{(\bullet)}\right)^{\ell} \text { with }|\ell|_{s} \geqslant p
\end{array}\right\}, \quad \text { for all } p \in \mathbb{N} .
$$

The graded terms $\operatorname{Gr}_{k-1}^{p}\left(E_{k, m}^{\mathrm{GG}} V^{*}\right)$, associated with the filtration $F_{k-1}^{p}\left(E_{k, m}^{\mathrm{GG}} V^{*}\right)$, are precisely the homogeneous polynomials $Q\left(f^{\prime}, f^{\prime \prime}, \ldots, f^{(k)}\right)$ all of whose monomials $\left(f^{(\bullet)}\right)^{\ell}$ have partial weighted degree $|\ell|_{k-1}=p$; hence, their degree $\ell_{k}$ in $f^{(k)}$ is such that $m-p=k \ell_{k}$ and $\operatorname{Gr}_{k-1}^{p}\left(E_{k, m}^{\mathrm{GG}} V^{*}\right)=0$ unless $k \mid m-p$. Looking at the transition automorphisms of the graded bundle induced by the coordinate change $f \mapsto \Psi \circ f$, it turns out that $f^{(k)}$ behaves as an element of $V \subset T_{X}$ and, as a simple computation shows, we find

$$
\mathrm{Gr}_{k-1}^{m-k \ell_{k}}\left(E_{k, m}^{\mathrm{GG}} V^{*}\right)=E_{k-1, m-k \ell_{k}}^{\mathrm{GG}} V^{*} \otimes S^{\ell_{k}} V^{*} .
$$




\section{DifFERENTIAL EQUATIONS ON COMPLEX PROJECTIVE HYPERSURFACES}

Combining all filtrations $F_{s}^{\bullet}$ together, we find inductively a filtration $F^{\bullet}$ on $E_{k, m}^{\mathrm{GG}} V^{*}$ such that the graded terms are

$$
\operatorname{Gr}^{\ell}\left(E_{k, m}^{\mathrm{GG}} V^{*}\right)=S^{\ell_{1}} V^{*} \otimes S^{\ell_{2}} V^{*} \otimes \cdots \otimes S^{\ell_{k}} V^{*}, \quad \ell \in \mathbb{N}^{k},|\ell|_{k}=m .
$$

Moreover there are natural induced filtrations $F_{s}^{p}\left(E_{k, m} V^{*}\right)=E_{k, m} V^{*} \cap F_{s}^{p}\left(E_{k, m}^{\mathrm{GG}} V^{*}\right)$ in such a way that

$$
\operatorname{Gr}^{\bullet}\left(E_{k, m} V^{*}\right)=\left(\bigoplus_{|\ell|_{k}=m} S^{\ell_{1}} V^{*} \otimes S^{\ell_{2}} V^{*} \otimes \cdots \otimes S^{\ell_{k}} V^{*}\right)^{\mathbb{G}_{k}^{\prime}}
$$

Here, we remark that, in general, it is a major unsolved problem to find the decomposition of $\operatorname{Gr}^{\bullet}\left(E_{k, m} V^{*}\right)$ into irreducible $\operatorname{Gl}\left(V^{*}\right)$-representations. This is easy for $k \leqslant 2$ (since the summands in the graded bundle do not mix up under the action of $\mathbb{G}_{k}^{\prime}$ ); [Rou06a] also found the formula for $k=\operatorname{dim} X=3$ and $V=T_{X}$, thanks to a deeper study based on a theorem of Popov on invariant theory.

\subsection{Projectivized $k$-jet bundles}

Here we explain the construction of the tower of projectivized bundles which provides a (relatively) smooth compactification of $J_{k}^{\mathrm{reg}} V / \mathbb{G}_{k}$, where $J_{k}^{\mathrm{reg}} V$ is the bundle of regular $k$-jets tangent to $V$, that is, $k$-jets such that $f^{\prime}(0) \neq 0$.

Let $(X, V)$ be a directed manifold, with $\operatorname{dim} X=n$ and $\operatorname{rank} V=r$. With $(X, V)$ we associate another directed manifold $(\widetilde{X}, \widetilde{V})$ where $\widetilde{X}=P(V)$ is the projectivized bundle of lines of $V, \pi: \widetilde{X} \rightarrow$ $X$ is the natural projection and $\widetilde{V}$ is the subbundle of $T_{\widetilde{X}}$ defined fibrewise as

$$
\widetilde{V}_{\left(x_{0},\left[v_{0}\right]\right)} \stackrel{\text { def }}{=}\left\{\xi \in T_{\widetilde{X},\left(x_{0},\left[v_{0}\right]\right)} \mid \pi_{*} \xi \in \mathbb{C} . v_{0}\right\},
$$

$x_{0} \in X$ and $v_{0} \in T_{X, x_{0}} \backslash\{0\}$. We also have a 'lifting' operator which assigns to a germ of holomorphic curve $f:(\mathbb{C}, 0) \rightarrow X$ tangent to $V$ a germ of holomorphic curve $\widetilde{f}:(\mathbb{C}, 0) \rightarrow \widetilde{X}$ tangent to $\widetilde{V}$ in such a way that $\widetilde{f}(t)=\left(f(t),\left[f^{\prime}(t)\right]\right)$.

To construct the projectivized $k$-jet bundle we simply set inductively $\left(X_{0}, V_{0}\right)=(X, V)$ and $\left(X_{k}, V_{k}\right)=\left(\widetilde{X}_{k-1}, \widetilde{V}_{k-1}\right)$. Of course, we have for each $k>0$ a tautological line bundle $\mathcal{O}_{X_{k}}(-1) \rightarrow$ $X_{k}$ and a natural projection $\pi_{k}: X_{k} \rightarrow X_{k-1}$. We call $\pi_{j, k}$ the composition of the projections $\pi_{j+1} \circ \cdots \circ \pi_{k}$, so that the total projection is given by $\pi_{0, k}: X_{k} \rightarrow X$. We have again for each $k>0$ short exact sequences

$$
\begin{gathered}
0 \rightarrow T_{X_{k} / X_{k-1}} \rightarrow V_{k} \rightarrow \mathcal{O}_{X_{k}}(-1) \rightarrow 0, \\
0 \rightarrow \mathcal{O}_{X_{k}} \rightarrow \pi_{k}^{*} V_{k-1} \otimes \mathcal{O}_{X_{k}}(1) \rightarrow T_{X_{k} / X_{k-1}} \rightarrow 0
\end{gathered}
$$

and $\operatorname{rank} V_{k}=r, \operatorname{dim} X_{k}=n+k(r-1)$. Here, we also have an inductively defined $k$-lifting for germs of holomorphic curves such that $f_{[k]}:(\mathbb{C}, 0) \rightarrow X_{k}$ is obtained as $f_{[k]}=\widetilde{f}_{[k-1]}$.

The following theorem justifies in some sense the construction of this projectivized bundle.

Theorem 3 [Dem97]. Suppose that $\operatorname{rank} V \geqslant 2$. Then the quotient $J_{k}^{\mathrm{reg}} V / \mathbb{G}_{k}$ has the structure of a locally trivial bundle over $X$, and there is a holomorphic embedding $J_{k}^{\mathrm{reg}} V / \mathbb{G}_{k} \hookrightarrow X_{k}$ over $X$, which identifies $J_{k}^{\mathrm{reg}} V / \mathbb{G}_{k}$ with $X_{k}^{\mathrm{reg}}$, that is the set of points in $X_{k}$ of the form $f_{[k]}(0)$ for some non-singular $k$-jet $f$. In other words $X_{k}$ is a relative compactification of $J_{k}^{\mathrm{reg}} V / \mathbb{G}_{k}$ over $X$.

Moreover, we have the direct image formula

$$
\left(\pi_{0, k}\right)_{*} \mathcal{O}_{X_{k}}(m)=\mathcal{O}\left(E_{k, m} V^{*}\right) .
$$

Next, we are in a position to point out the link between the theory of hyperbolicity and invariant jet differentials. 


\section{S. DIVERIO}

Theorem 4 [GG79, Dem97]. Assume that there exist integers $k, m>0$ and an ample line bundle $A \rightarrow X$ such that

$$
H^{0}\left(X_{k}, \mathcal{O}_{X_{k}}(m) \otimes \pi_{0, k}^{*} A^{-1}\right) \simeq H^{0}\left(X, E_{k, m} V^{*} \otimes A^{-1}\right)
$$

has non-zero sections $\sigma_{1}, \ldots, \sigma_{N}$ and let $Z \subset X_{k}$ be the base locus of these sections. Then every entire holomorphic curve $f: \mathbb{C} \rightarrow X$ tangent to $V$ is such that $f_{[k]}(\mathbb{C}) \subset Z$. In other words, for every global $\mathbb{G}_{k}$-invariant differential equation $P$ vanishing on an ample divisor, every entire holomorphic curve $f$ must satisfy the algebraic differential equation $P(f)=0$ (and a similar result is true also for the bundle $E_{k, m}^{\mathrm{GG}} T_{X}^{*}$ ).

\subsection{Algebraic holomorphic Morse inequalities}

Let $L \rightarrow X$ be a holomorphic line bundle over a compact complex manifold of dimension $n$ and $E \rightarrow X$ a holomorphic vector bundle of rank $r$. Suppose that $L$ can be written as the difference of two numerically effective (nef) line bundles, say $L=F \otimes G^{-1}$, with $F, G \rightarrow X$ numerically effective. Then we have the following asymptotic estimate for the partial alternating sum of the dimension of cohomology groups of $L$ with values in $E$.

Theorem 5 [Dem01]. With the previous notation, we have (strong algebraic holomorphic Morse inequalities):

$$
\sum_{j=0}^{q}(-1)^{q-j} h^{j}\left(X, L^{\otimes m} \otimes E\right) \leqslant r \frac{m^{n}}{n !} \sum_{j=0}^{q}(-1)^{q-j}\left(\begin{array}{c}
n \\
j
\end{array}\right) F^{n-j} \cdot G^{j}+o\left(m^{n}\right) .
$$

In particular [Tra95], $L^{\otimes m} \otimes E$ has a global section for $m$ large if $F^{n}-n F^{n-1} \cdot G>0$.

\subsection{Schur powers of a complex vector space}

Here, we just recall the notation and a possible construction of Schur powers of a complex vector space. Let $V$ be a complex vector space of dimension $r$. With every set of non-increasing $r$-tuples $\left(a_{1}, \ldots, a_{r}\right) \in \mathbb{Z}^{r}, a_{1} \geqslant a_{2} \geqslant \cdots \geqslant a_{r}$, one associates, in a functorial way, a collection of vector spaces $\Gamma^{\left(a_{1}, \ldots, a_{r}\right)} V$, which provide the list of all irreducible representations of the linear group $\operatorname{Gl}(V)$, up to isomorphism (in fact $\left(a_{1}, \ldots, a_{r}\right)$ is the highest weight of the action of a maximal torus $\left.\left(\mathbb{C}^{*}\right)^{r} \subset \mathrm{Gl}(V)\right)$. The Schur functors can be defined in an elementary way as follows. Let

$$
\mathbb{U}_{r}=\left\{\left(\begin{array}{ll}
1 & 0 \\
* & 1
\end{array}\right)\right\}
$$

be the group of lower triangular unipotent $r \times r$ matrices. If all $a_{j}$ are non-negative, one defines

$$
\Gamma^{\left(a_{1}, \ldots, a_{r}\right)} V \subset S^{a_{1}} \otimes \cdots \otimes S^{a_{r}} V
$$

as the set of polynomials $P\left(\xi_{1}, \ldots, \xi_{r}\right)$ on $\left(V^{*}\right)^{r}$ which are homogeneous of degree $a_{j}$ with respect to $\xi_{j}$ and which are invariant under the left action of $\mathbb{U}_{r}$ on $\left(V^{*}\right)^{r}=\operatorname{Hom}\left(V, \mathbb{C}^{r}\right)$, i.e.

$$
P\left(\xi_{1}, \ldots, \xi_{j-1}, \xi_{j}+\xi_{k}, \xi_{j+1}, \ldots, \xi_{r}\right)=P\left(\xi_{1}, \ldots, \xi_{r}\right), \quad \text { for all } k<j .
$$

We agree that $\Gamma^{\left(a_{1}, \ldots, a_{r}\right)} V=0$ unless $\left(a_{1}, \ldots, a_{r}\right)$ is non-increasing. As a special case, we recover symmetric and exterior powers

$$
\begin{aligned}
& S^{m} V=\Gamma^{(m, 0, \ldots, 0)} V, \\
& \bigwedge^{k} V=\Gamma^{(1, \ldots, 1,0, \ldots, 0)} V, \quad \text { with } k \text { indices } 1 .
\end{aligned}
$$

The Schur functors satisfy the well known formula

$$
\Gamma^{\left(a_{1}+\ell, \ldots, a_{r}+\ell\right)} V=\Gamma^{\left(a_{1}, \ldots, a_{r}\right)} V \otimes(\operatorname{det} V)^{\ell},
$$

which can be used to define $\Gamma^{\left(a_{1}, \ldots, a_{r}\right)} V$ if any of the $a_{j}$ happens to be negative. 


\section{DifFERENTIAL EQUATIONS ON COMPLEX PROJECTIVE HYPERSURFACES}

\section{Proof of Theorem 1}

First of all, we recall a theorem contained in [BR90].

Theorem 6. Let $X$ be a $n$-dimensional smooth complete intersection in $\mathbb{P}^{N}$. Let $T$ be any Young tableau and $t_{i}$ be the number of cells inside the ith column of T. Set

$$
t:=\sum_{i=1}^{N-n} t_{i}, \quad t_{i}=0 \text { if } i>\operatorname{length} T .
$$

Then, if $t<n$ one has the vanishing

$$
H^{0}\left(X, \Gamma^{T} T_{X}^{*}\right)=0,
$$

i.e. the smooth complete intersection $X$ has no global $T$-symmetrical tensor forms different from zero if the Young tableau $T$ has less than $\operatorname{dim} X$ cells inside its codim $X$ front columns.

In our notation, the irreducible $\mathrm{Gl}\left(T_{X}^{*}\right)$-representation, given for $\lambda_{1} \geqslant \lambda_{2} \geqslant \cdots \geqslant \lambda_{n}$ by $\Gamma^{\left(\lambda_{1}, \ldots, \lambda_{n}\right)} T_{X}^{*}$, corresponds to $\Gamma^{T_{\lambda}} T_{X}^{*}$ where the tableau $T_{\lambda}$ is obtained from the partition $\lambda_{1}+\cdots+\lambda_{n}$. Thus, for example, the tableau with only one row of length $m$ corresponds to $S^{m} T_{X}^{*}$ and the tableau with only one column of depth $k$ corresponds to $\bigwedge^{k} T_{X}^{*}$.

We now need an algebraic lemma.

Lemma 1. Let $V$ be a complex vector space of dimension $n$ and $\lambda=\left(\lambda_{1}, \ldots, \lambda_{n}\right)$ such that $\lambda_{1} \geqslant$ $\lambda_{2} \geqslant \cdots \geqslant \lambda_{n} \geqslant 0$. Then

$$
\Gamma^{\lambda} V \otimes S^{m} V \simeq \bigoplus_{\mu} \Gamma^{\mu} V
$$

as $\mathrm{Gl}(V)$-representations, the sum being over all $\mu$ whose Young diagram is obtained by adding $m$ boxes to the Young diagram of $\lambda$, with no two in the same column.

Proof. This follows immediately by Pieri's formula; see, e.g., [FH91].

Note that this implies that among the irreducible $\operatorname{Gl}(V)$-representations of $S^{l} V \otimes S^{m} V$, we cannot find terms of type $\Gamma^{\left(\lambda_{1}, \ldots, \lambda_{n}\right)} V$ with $\lambda_{i}>0$ for $i>2$ (they are all of type $\Gamma^{(l+m-j, j, 0, \ldots, 0)} V$ for $j=0, \ldots, \min \{m, l\})$.

Thus, by induction on the number of factors in the tensor product of symmetric powers, we easily find the following result.

Proposition 1. If $k \leqslant n$, then we have a direct sum decomposition into irreducible $\operatorname{Gl}(V)$ representations

$$
S^{l_{1}} V \otimes S^{l_{2}} V \otimes \cdots \otimes S^{l_{k}} V=\bigoplus_{\lambda} \nu_{\lambda} \Gamma^{\lambda} V
$$

where $\nu_{\lambda} \neq 0$ only if $\lambda=\left(\lambda_{1}, \ldots, \lambda_{n}\right)$ is such that $\lambda_{i}=0$ for $i>k$.

Now, we can state and prove a slightly more general version (which actually implies immediately Theorem 1) of our first result.

Theorem 7. Let $X \subset \mathbb{P}^{N}$ be a smooth complete intersection. Then

$$
H^{0}\left(X, E_{k, m}^{\mathrm{GG}} T_{X}^{*}\right)=0
$$

for all $m \geqslant 1$ and $1 \leqslant k<\operatorname{dim} X / \operatorname{codim} X$. 


\section{S. DIVERIO}

Proof. The bundle $E_{k, m}^{\mathrm{GG}} T_{X}^{*}$ admits a filtration whose associated graded bundle is given by

$$
\mathrm{Gr}^{\bullet} E_{k, m}^{\mathrm{GG}} T_{X}^{*}=\bigoplus_{l_{1}+2 l_{2}+\cdots+k l_{k}=m} S^{l_{1}} T_{X}^{*} \otimes S^{l_{2}} T_{X}^{*} \otimes \cdots \otimes S^{l_{k}} T_{X}^{*} .
$$

The addenda in the direct sum decomposition into irreducible $\mathrm{Gl}\left(T_{X}^{*}\right)$-representation of $\mathrm{Gr}^{\bullet} E_{k, m}^{\mathrm{GG}} T_{X}^{*}$ are all of type $\Gamma^{\left(\lambda_{1}, \ldots, \lambda_{n}\right)} T_{X}^{*}$ with $\lambda_{i}=0$ for $i>k$. This means that, in the statement of Theorem 6 , each $t_{i}$ is less than or equal to $k$. Then, if $\operatorname{dim} X=n$, we have

$$
\begin{aligned}
t & =\sum_{i=1}^{N-n} t_{i} \leqslant \sum_{i=1}^{N-n} k \\
& =k(N-n)<\frac{n}{N-n}(N-n)=n .
\end{aligned}
$$

Thus, by Theorem 6, we have the vanishing of the global section of each graded piece. We now only need to link the vanishing of the cohomology of a filtered vector bundle to the vanishing of its graded bundle. This is done in the next lemma and the proof is achieved.

Lemma 2. Let $E \rightarrow X$ be a holomorphic filtered vector bundle with filtered pieces $\{0\}=E_{r} \subset$ $\cdots \subset E_{p+1} \subset E_{p} \subset \cdots \subset E_{0}=E$. If $H^{q}\left(X, \operatorname{Gr}^{\bullet} E\right)=0$ then $H^{q}(X, E)=0$.

Proof. Consider the short exact sequence

$$
0 \rightarrow \mathrm{Gr}^{p} E \rightarrow E / E_{p+1} \rightarrow E / E_{p} \rightarrow 0
$$

and the associated long exact sequence in cohomology

$$
\cdots \rightarrow \underbrace{H^{q}\left(X, \mathrm{Gr}^{p} E\right)}_{=0} \rightarrow H^{q}\left(X, E / E_{p+1}\right) \rightarrow H^{q}\left(X, E / E_{p}\right) \rightarrow \cdots
$$

For $p=1$, we get $H^{q}\left(X, E / E_{1}\right)=H^{q}\left(X, \operatorname{Gr}^{0} E\right)=0$ by hypothesis. Thus, $H^{q}\left(X, E / E_{2}\right)=0$ and, by induction on $p$, we find $H^{q}\left(X, E / E_{p}\right)=0$. Therefore, for $p=r$ we get the desired result.

\section{Proof of Theorem 2}

In this section, we prove our Theorem 2 by means of the algebraic version of holomorphic Morse inequalities, performed on a particular subbundle of the invariant jet differentials. For the sake of simplicity, we shall reproduce here only the proof for three jets on threefolds in $\mathbb{P}^{4}$, the other cases being completely analogous.

\subsection{Chern class computations}

Denote by $c_{\bullet}(E)$ the total Chern class of a vector bundle $E$. The short exact sequences $(1)$ and (2) give us, for each $k>0$, the following formulae:

$$
c_{\bullet}\left(V_{k}\right)=c_{\bullet}\left(T_{X_{k} / X_{k-1}}\right) c_{\bullet}\left(\mathcal{O}_{X_{k}}(-1)\right)
$$

and

so that

$$
c_{\bullet}\left(\pi_{k}^{*} V_{k-1} \otimes \mathcal{O}_{X_{k}}(1)\right)=c_{\bullet}\left(T_{X_{k} / X_{k-1}}\right),
$$

$$
c_{\bullet}\left(V_{k}\right)=c_{\bullet}\left(\mathcal{O}_{X_{k}}(-1)\right) c_{\bullet}\left(\pi_{k}^{*} V_{k-1} \otimes \mathcal{O}_{X_{k}}(1)\right) .
$$

Let us call $u_{j}=c_{1}\left(\mathcal{O}_{X_{j}}(1)\right)$ and $c_{l}^{[j]}=c_{l}\left(V_{j}\right)$. With this notation, (3) becomes

$$
1+c_{1}^{[k]}+\cdots+c_{r}^{[k]}=\left(1-u_{k}\right) \sum_{0 \leqslant j \leqslant r} \pi_{k}^{*} c_{j}^{[k-1]}\left(1+u_{k}\right)^{r-j} .
$$




\section{DIFFERENTIAL EQUATIONS ON COMPLEX PROJECTIVE HYPERSURFACES}

Since $X_{j}$ is the projectivized bundle of line of $V_{j-1}$, we also have the polynomial relations

$$
u_{j}^{r}+\pi_{j}^{*} c_{1}^{[j-1]} \cdot u_{j}^{r-1}+\cdots+\pi_{j}^{*} c_{r-1}^{[j-1]} \cdot u_{j}+\pi_{j}^{*} c_{r}^{[j-1]}=0, \quad 1 \leqslant j \leqslant k .
$$

Proposition 2. Let $\operatorname{rank} V=3$. Then, we have the following relations for Chern classes:

$$
\begin{aligned}
& c_{1}^{[k]}=\pi_{k}^{*} c_{1}^{[k-1]}+2 u_{k}, \\
& c_{2}^{[k]}=\pi_{k}^{*} c_{2}^{[k-1]}+\pi_{k}^{*} c_{1}^{[k-1]} \cdot u_{k}, \\
& c_{3}^{[k]}=\pi_{k}^{*} c_{3}^{[k-1]}-\pi_{k}^{*} c_{1}^{[k-1]} \cdot u_{k}^{2}-2 u_{k}^{3}, \\
& u_{k}^{3}+\pi_{k}^{*} c_{1}^{[k-1]} \cdot u_{j}^{2}+\pi_{k}^{*} c_{2}^{[k-1]} \cdot u_{k}+\pi_{k}^{*} c_{3}^{[k-1]}=0 .
\end{aligned}
$$

Proof. This is just a straightforward computation using identity (4).

Next, let $X \subset \mathbb{P}^{n+1}$ be a smooth hypersurface of degree $\operatorname{deg} X=d$. Then, we have a short exact sequence

$$
\left.0 \rightarrow T_{X} \rightarrow T_{\mathbb{P}^{n+1}}\right|_{X} \rightarrow \mathcal{O}_{X}(d) \rightarrow 0 .
$$

So we have the following relation for the total Chern class of $X$ :

$$
(1+h)^{n+2}=(1+d h) c_{\bullet}(X),
$$

where $h=c_{1}\left(\mathcal{O}_{\mathbb{P}^{n+1}}(1)\right)$ and $(1+h)^{n+2}$ is the total Chern class of $\mathbb{P}^{n+1}$. Thus, an easy computation yields the next result.

Proposition 3. Let $X \subset \mathbb{P}^{4}$ be a smooth hypersurface of degree $\operatorname{deg} X=d$. Then, the Chern classes of $X$ are given (in terms of the hyperplane class) by

$$
\begin{aligned}
& c_{1}(X)=-h(d-5), \\
& c_{2}(X)=h^{2}\left(d^{2}-5 d+10\right), \\
& c_{3}(X)=-h^{3}\left(d^{3}-5 d^{2}+10 d-10\right)
\end{aligned}
$$

and $h^{3}=d$.

\subsection{Choice of the appropriate subbundle}

By definition, there is a canonical injection $\mathcal{O}_{X_{k}}(-1) \hookrightarrow \pi_{k}^{*} V_{k-1}$, and a composition with the differential of the projection $\left(\pi_{k}\right)_{*}$ yields, for all $k \geqslant 2$, a canonical line bundle morphism

$$
\mathcal{O}_{X_{k}}(-1) \hookrightarrow \pi_{k}^{*} V_{k-1} \rightarrow \pi_{k}^{*} \mathcal{O}_{X_{k-1}}(-1),
$$

which admits precisely $D_{k} \stackrel{\text { def }}{=} P\left(T_{X_{k-1} / X_{k-2}}\right) \subset P\left(V_{k-1}\right)=X_{k}$ as its zero divisor. Hence, we find

$$
\mathcal{O}_{X_{k}}(1)=\pi_{k}^{*} \mathcal{O}_{X_{k-1}}(1) \otimes \mathcal{O}\left(D_{k}\right) .
$$

Now, for $\mathbf{a}=\left(a_{1}, \ldots, a_{k}\right) \in \mathbb{Z}^{k}$, define a line bundle $\mathcal{O}_{X_{k}}(\mathbf{a})$ on $X_{k}$ as

$$
\mathcal{O}_{X_{k}}(\mathbf{a})=\pi_{1, k}^{*} \mathcal{O}_{X_{1}}\left(a_{1}\right) \otimes \pi_{2, k}^{*} \mathcal{O}_{X_{2}}\left(a_{2}\right) \otimes \cdots \otimes \mathcal{O}_{X_{k}}\left(a_{k}\right) .
$$

By (8), we have

$$
\pi_{j, k}^{*} \mathcal{O}_{X_{j}}(1)=\mathcal{O}_{X_{k}}(1) \otimes \mathcal{O}_{X_{k}}\left(-\pi_{j+1, k}^{*} D_{j+1}-\cdots-D_{k}\right),
$$

and thus, by setting $D_{j}^{\star}=\pi_{j+1, k}^{*} D_{j+1}$ for $j=1, \ldots, k-1$ and $D_{k}^{\star}=0$, we have an identity

$$
\mathcal{O}_{X_{k}}(\mathbf{a})=\mathcal{O}_{X_{k}}\left(b_{k}\right) \otimes \mathcal{O}_{X_{k}}\left(-\mathbf{b} \cdot D^{\star}\right)
$$




\section{S. DIVERIO}

where

$$
\begin{aligned}
\mathbf{b} & =\left(b_{1}, \ldots, b_{k}\right) \in \mathbb{Z}^{k}, \quad b_{j}=a_{1}+\cdots+a_{j}, \\
\mathbf{b} \cdot D^{\star} & =\sum_{j=1}^{k-1} b_{j} \pi_{j+1, k}^{*} D_{j+1} .
\end{aligned}
$$

In particular, if $\mathbf{b} \in \mathbb{N}^{k}$, that is if $a_{1}+\cdots+a_{j} \geqslant 0$, we get a morphism

$$
\mathcal{O}_{X_{k}}(\mathbf{a})=\mathcal{O}_{X_{k}}\left(b_{k}\right) \otimes \mathcal{O}_{X_{k}}\left(-\mathbf{b} \cdot D^{\star}\right) \rightarrow \mathcal{O}_{X_{k}}\left(b_{k}\right) .
$$

We then have the following proposition.

Proposition 4 [Dem97]. Let $\mathbf{a}=\left(a_{1}, \ldots, a_{k}\right) \in \mathbb{N}^{k}$ and $m=a_{1}+\cdots+a_{k}$.

(a) We have the direct image formula

$$
\left(\pi_{0, k}\right)_{*} \mathcal{O}_{X_{k}}(\mathbf{a}) \simeq \mathcal{O}\left(\bar{F}^{\mathbf{a}} E_{k, m} V^{*}\right) \subset \mathcal{O}\left(E_{k, m} V^{*}\right)
$$

where $\bar{F}^{\mathbf{a}} E_{k, m} V^{*}$ is the subbundle of polynomials $Q\left(f^{\prime}, f^{\prime \prime}, \ldots, f^{(k)}\right) \in E_{k, m} V^{*}$ involving only monomials $\left(f^{(\bullet)}\right)^{\ell}$ such that

$$
\ell_{s+1}+2 \ell_{s+2}+\cdots+(k-s) \ell_{k} \leqslant a_{s+1}+\cdots+a_{k}
$$

for all $s=0, \ldots, k-1$.

(b) If $a_{1} \geqslant 3 a_{2}, \ldots, a_{k-2} \geqslant 3 a_{k-1}$ and $a_{k-1} \geqslant 2 a_{k}>0$, the line bundle $\mathcal{O}_{X_{k}}(\mathbf{a})$ is relatively nef over $X$.

In particular, the line bundle $\mathcal{L}_{k}(X) \stackrel{\text { def }}{=} \mathcal{O}_{X_{k}}\left(2 \cdot 3^{k-2}, 2 \cdot 3^{k-3}, \ldots, 6,2,1\right)$ is relatively nef over $X$ and its direct image on $X$ is a subbundle of the bundle of invariant jet differentials of order $k$ and weighted degree $3^{k-1}$.

In the case of projective hypersurface, we obtain the following expression of $\mathcal{L}_{k}$ as the difference of two nef line bundles.

Lemma 3. Let $X \subset \mathbb{P}^{n+1}$ be a projective hypersurface. Then $\mathcal{L}_{k}(X) \otimes \pi_{0, k}^{*} \mathcal{O}_{X}(l)$ is nef if $l \geqslant 2 \cdot 3^{k-1}$. In particular,

$$
\mathcal{L}_{k}(X)=\mathcal{F}_{k}(X) \otimes \mathcal{G}_{k}(X)^{-1},
$$

where $\mathcal{F}_{k}(X)=\mathcal{L}_{k}(X) \otimes \pi_{0, k}^{*} \mathcal{O}_{X}\left(2 \cdot 3^{k-1}\right)$ and $\mathcal{G}_{k}(X)=\pi_{0, k}^{*} \mathcal{O}_{X}\left(2 \cdot 3^{k-1}\right)$ are nef.

Proof. Of course, as a pull-back of an ample line bundle,

$$
\mathcal{G}_{k}(X)=\pi_{0, k}^{*} \mathcal{O}_{X}\left(2 \cdot 3^{k-1}\right)
$$

is nef. It is well known that the cotangent space of the projective space twisted by $\mathcal{O}(2)$ is globally generated. Hence, $T_{X}^{*} \otimes \mathcal{O}_{X}(2)$ is globally generated as a quotient of $\left.T_{\mathbb{P}^{n+1}}^{*}\right|_{X} \otimes \mathcal{O}_{X}(2)$, so that $\mathcal{O}_{X_{1}}(1) \otimes \pi_{0,1}^{*} \mathcal{O}_{X}(2)=\mathcal{O}_{\mathbb{P}\left(T_{X}^{*} \otimes \mathcal{O}_{X}(2)\right)}(1)$ is nef.

Next, we construct, by induction on $k$, a nef line bundle $A_{k} \rightarrow X_{k}$ such that $\mathcal{O}_{X_{k+1}}(1) \otimes \pi_{k}^{*} A_{k}$ is nef. By definition, this is equivalent to saying that the vector bundle $V_{k}^{*} \otimes A_{k}$ is nef. By what we have just seen, we can take $A_{0}=\mathcal{O}_{X}(2)$ on $X_{0}=X$. Suppose $A_{0}, \ldots, A_{k-1}$ have been constructed. As an extension of nef vector bundles is nef, dualizing the short exact sequence (1) we find

$$
0 \rightarrow \mathcal{O}_{X_{k}}(1) \rightarrow V_{k}^{*} \rightarrow T_{X_{k} / X_{k-1}}^{*} \rightarrow 0,
$$

and so we see, twisting by $A_{k}$, that it suffices to select $A_{k}$ in such a way that both $\mathcal{O}_{X_{k}}(1) \otimes A_{k}$ and $T_{X_{k} / X_{k-1}}^{*} \otimes A_{k}$ are nef. To this aim, considering the second wedge power of the central term in (2), we get an injection

$$
0 \rightarrow T_{X_{k} / X_{k-1}} \rightarrow \bigwedge^{2}\left(\pi_{k}^{*} V_{k-1} \otimes \mathcal{O}_{X_{k}}(1)\right)
$$




\section{DifFERENTIAL EQUATIONS ON COMPLEX PROJECTIVE HYPERSURFACES}

and so dualizing and twisting by $\mathcal{O}_{X_{k}}(2) \otimes \pi_{k}^{*} A_{k-1}^{\otimes 2}$, we find a surjection

$$
\pi_{k}^{*} \bigwedge^{2}\left(V_{k-1}^{*} \otimes A_{k-1}\right) \rightarrow T_{X_{k} / X_{k-1}}^{*} \otimes \mathcal{O}_{X_{k}}(2) \otimes \pi_{k}^{*} A_{k-1}^{\otimes 2} \rightarrow 0 .
$$

By induction hypothesis, $V_{k-1}^{*} \otimes A_{k-1}$ is nef so the quotient $T_{X_{k} / X_{k-1}}^{*} \otimes \mathcal{O}_{X_{k}}(2) \otimes \pi_{k}^{*} A_{k-1}^{\otimes 2}$ is nef, too. In order to have the nefness of both $\mathcal{O}_{X_{k}}(1) \otimes A_{k}$ and $T_{X_{k} / X_{k-1}}^{*} \otimes A_{k}$, it is enough to choose $A_{k}$ in such a way that $A_{k} \otimes \pi_{k}^{*} A_{k-1}^{*}$ and $A_{k} \otimes \mathcal{O}_{X_{k}}(-2) \otimes \pi_{k}^{*} A_{k-1}^{* \otimes 2}$ are both nef: therefore we set

$$
A_{k}=\mathcal{O}_{X_{k}}(2) \otimes \pi_{k}^{*} A_{k-1}^{\otimes 3}=\left(\mathcal{O}_{X_{k}}(1) \otimes \pi_{k}^{*} A_{k-1}\right)^{\otimes 2} \otimes \pi_{k}^{*} A_{k-1},
$$

which, as a product of nef line bundles, is nef and satisfies the two conditions above. This gives $A_{k}$ inductively, and the resulting formula for $\mathcal{O}_{X_{k}}(1) \otimes \pi_{k}^{*} A_{k-1}$ is

$$
\begin{aligned}
\mathcal{O}_{X_{k}}(1) \otimes \pi_{k}^{*} A_{k-1} & =\mathcal{L}_{k}(X) \otimes \pi_{0, k}^{*} \mathcal{O}_{X}\left(2 \cdot\left(1+2+\cdots+2 \cdot 3^{k-2}\right)\right) \\
& =\mathcal{L}_{k}(X) \otimes \pi_{0, k}^{*} \mathcal{O}_{X}\left(2 \cdot 3^{k-1}\right) .
\end{aligned}
$$

The lemma is proved.

\subsection{End of the proof of Theorem 2}

We just apply the algebraic version of holomorphic Morse inequalities to the line bundle $\mathcal{L}_{3}(X)$ for $X$ a smooth hypersurface in $\mathbb{P}^{4}$ (and, for the other cases, to the line bundle $\mathcal{L}_{n}(X)$ for $X$ a smooth hypersurface in $\left.\mathbb{P}^{n+1}, n=2,4,5\right)$.

Then, we have to compute $\mathcal{F}_{3}(X)^{9}-9 \mathcal{F}_{3}(X)^{8} \cdot \mathcal{G}_{3}(X)$ which is given in terms of Chern classes by

$$
\left(u_{3}+2 \pi_{2,3}^{*} u_{2}+6 \pi_{1,3}^{*} u_{1}+18 \pi_{0,3}^{*} h\right)^{9}-9\left(u_{3}+2 \pi_{2,3}^{*} u_{2}+6 \pi_{1,3}^{*} u_{1}+18 \pi_{0,3}^{*} h\right)^{8} \cdot 18 \pi_{0,3}^{*} h .
$$

By using Proposition 2 recursively we can express this quantity in terms of Chern classes of $X$ and the hyperplane class (the computation is made with GP/PARI CALCULATOR Version 2.3.2):

$$
\begin{aligned}
& -3421377792 h^{3}+676045440 c_{1}(X) \cdot h^{2}-7494966 c_{1}(X)^{3} \\
& +10997352 c_{2}(X) \cdot c_{1}(X)-3835548 c_{3}(X),
\end{aligned}
$$

and, by Proposition 3, we obtain

$$
\mathcal{F}_{3}(X)^{9}-9 \mathcal{F}_{3}(X)^{8} \cdot \mathcal{G}_{3}(X)=333162 d^{4}-21628710 d^{3}-460474830 d^{2}-466509222 d,
$$

which is positive if $d=\operatorname{deg}(X) \geqslant 82$.

Remark 1. Even if we know [Rou06b] that the line bundle $\mathcal{O}_{X_{3}}(1)$ is big in the case of smooth hypersurfaces in projective 4 -space for $\operatorname{deg}(X) \geqslant 97$, to get the result with these techniques we are obliged to deal with the line bundle $\mathcal{L}_{3}(X)$. In fact, the algebraic version of holomorphic Morse inequalities gives, if we merely utilize $\mathcal{O}_{X_{3}}(1)$, a negative lower bound (and similarly in higher dimension). The reason is that $\mathcal{O}_{X_{k}}(1)$ is always relatively big but never relatively nef (if $k \geqslant 2$ ) so that with $\mathcal{O}_{X_{k}}(1)$ holomorphic Morse inequalities take into account too many 'relatively negative terms'.

Remark 2. With a slight theoretical refinement of these techniques, we are able to show the existence of global invariant jet differentials vanishing on an ample divisor even for the dimension of the smooth hypersurface less than or equal to 8. Unfortunately, the trick we use to increase the dimension leads to the loss of the effectivity: we can show the existence of a lower bound for the degree depending only on the dimension of the hypersurface, but we are unable to compute it.

However, we feel strongly that in the forthcoming future, with further improvement in the comprehension of the cohomology algebra of $X_{k}$, we will be able to show (without explicit effectivity) the existence of global invariant jet differentials vanishing on an ample divisor for smooth hypersurfaces of any dimension. 


\section{S. DIVERIO}

\section{Computer calculations}

Here we reproduce the code we utilized to perform all computations with GP/PARI CALCULATOR Version 2.3.2.

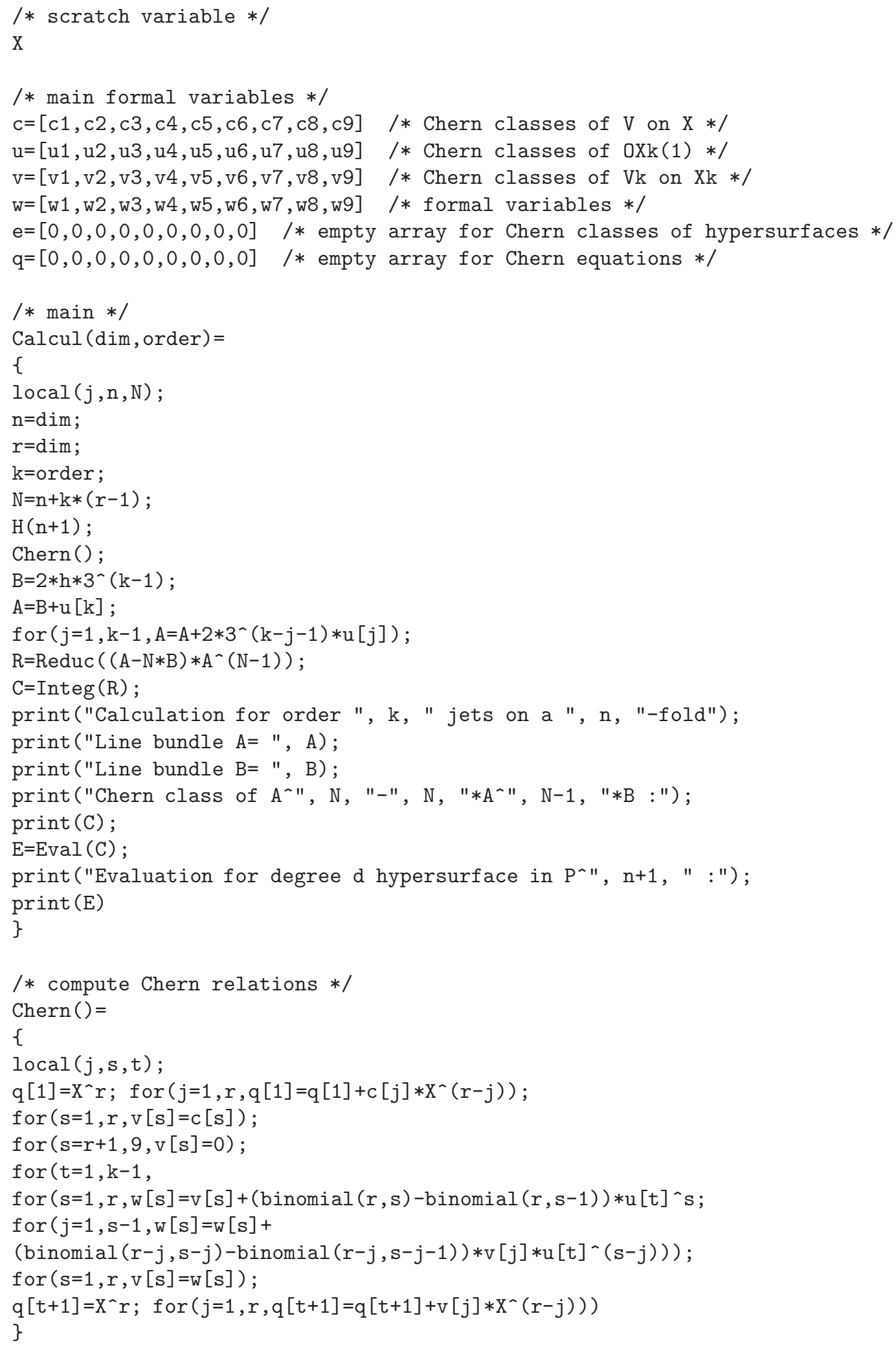




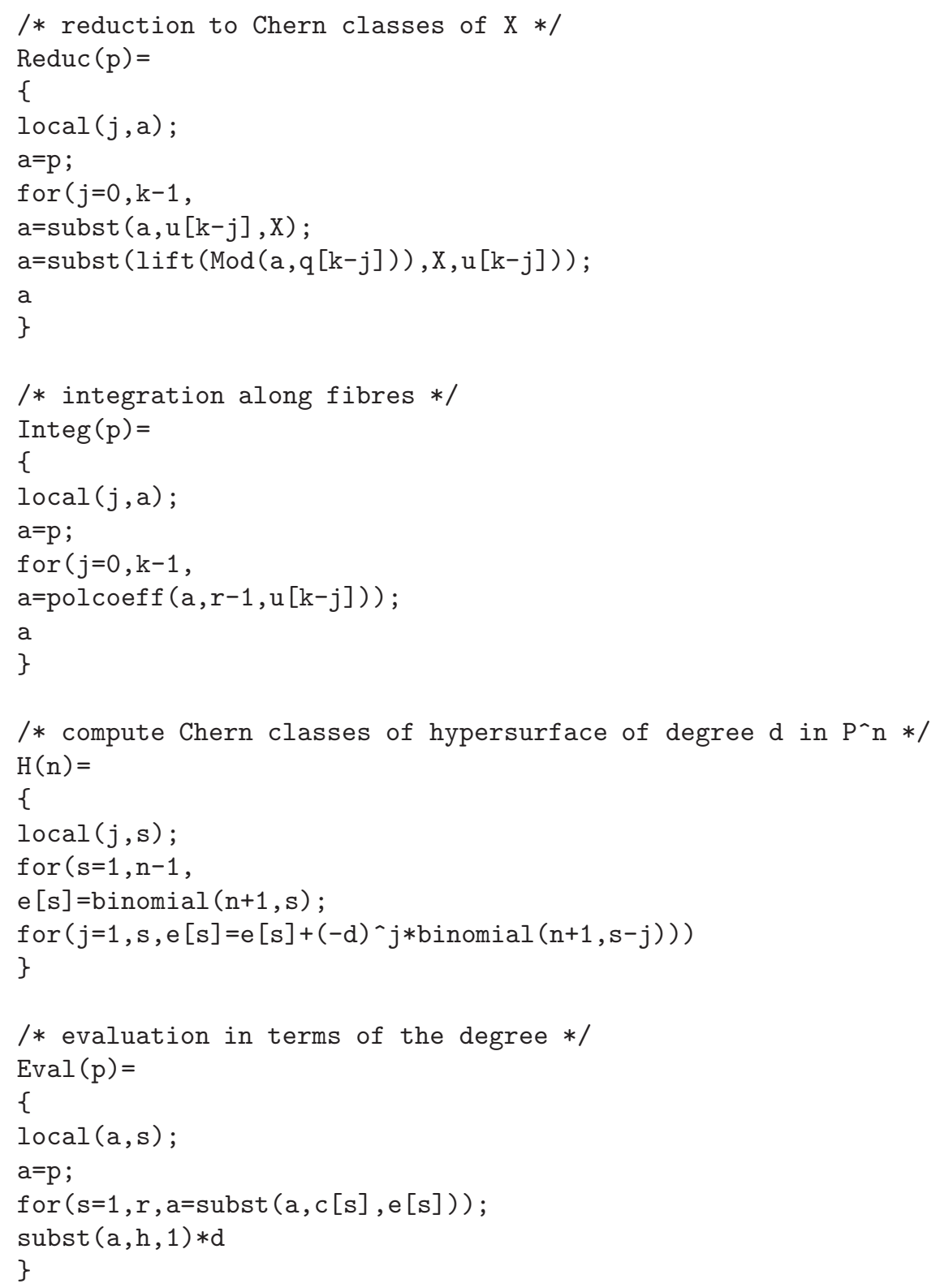

\section{ACKNOWLEDGEMENTS}

I would like to thank my two thesis directors, Professor Jean-Pierre Demailly and Professor Stefano Trapani, for their help, encouragement, extreme patience and nice attitude; and of course for all the formal talks and informal chats we had which introduced me to this subject. Thanks also go to Andrea Maffei for his 'algebraic support' and to Erwan Rousseau for having generously shared with me some of his ideas about the vanishing of jet differentials.

\section{REFERENCES}

BR90 P. Brückmann and H.-G. Rackwitz, T-symmetrical tensor forms on complete intersections, Math. Ann. 288 (1990), 627-635.

Dem97 J.-P. Demailly, Algebraic criteria for kobayashi hyperbolic projective varieties and jet differentials, in Algebraic geometry, Santa Cruz, 1995, Proceedings of Symposia in Pure Mathematics, vol. 62, part 2 (American Mathematical Society, Providence, RI, 1997), 285-360. 


\section{DifFERENTIAL EQUATIONS ON COMPLEX PROJECTIVE HYPERSURFACES}

Dem01 J.-P. Demailly, Multiplier ideal sheaves and analytic methods in algebraic geometry, in School on vanishing theorems and effective results in algebraic geometry, Trieste, 2000, ICTP Lecture Notes, vol. 5 (Abdus Salam International Center for Theoretical Physics, Trieste, 2001), 1-148.

DEG00 J.-P. Demailly and J. El Goul, Hyperbolicity of generic surfaces of high degree in projective 3-space, Amer. J. Math 122 (2000), 515-546.

FH91 W. Fulton and J. Harris, Representation theory: a first course, Graduate Texts in Mathematics, vol. 129, Readings in Mathematics (Springer, New York, 1991).

GG79 M. Green and P. Griffiths, Two applications of algebraic geometry to entire holomorphic mappings, in The Chern Symposium 1979, Proc. Internat. Sympos., Berkeley, CA, 1979 (Springer, New York, 1980), 41-74.

Kob70 S. Kobayashi, Hyperbolic manifolds and holomorphic mappings (Marcel Dekker, New York, 1970).

Rou07 E. Rousseau, Weak analytic hyperbolicity of generic hypersurfaces of high degree in $\mathbb{P}^{4}$, Ann. Fac. Sci. Toulouse Math. (6) 16 (2007).

Rou06a E. Rousseau, Étude des jets de Demailly-Semple en dimension 3, Ann. Inst. Fourier (Grenoble) 56 (2006), 397-421.

Rou06b E. Rousseau, Équations différentielles sur les hypersurfaces de $\mathbb{P}^{4}$, J. Math. Pures Appl. (9) 86 (2006), 322-341.

Siu04 Y.-T. Siu, Hyperbolicity in complex geometry, in The legacy of Niel Henrik Abel (Springer, Berlin, 2004), 543-566.

Tra95 S. Trapani, Numerical criteria for the positivity of the difference of ample divisors, Math. Z. 219 (1995), 387-401.

Simone Diverio sdiverio@fourier.ujf-grenoble.fr

Institut Fourier, Université de Grenoble I, BP 74, F-38402, Saint Martin d'Héres, France

Current address: Istituto 'Guido Castelnuovo', Università di Roma 'La Sapienza',

P. le Aldo Moro 2, 00185 Roma, Italy 